\title{
ON THE DISCRIMINANT OF TERNARY FORMS AND A CERTAIN CLASS OF SURFACES*
}

\author{
BY \\ ARNOLD EMCH
}

I. INTRODUCTION

In a paper On the Weddle surface and some analogous loci $\dagger \mathrm{I}$ have studied the class of surfaces and curves defined as the loci of vertices of general cones passing through certain definite numbers of generic fixed points in space.

This investigation may be extended to such systems of cones with nodal generatrices. For this purpose it is obviously necessary to make use of the properties of the discriminant of a ternary form, to which the intersection of a cone with a generic plane reduces.

\section{THE DISCRIMINANT OF A TERNARY FORM}

Let

$$
C_{n}=\sum a_{\alpha \beta \gamma} X^{\alpha} Y^{\beta} Z^{\gamma}=0, \quad \alpha+\beta+\gamma=n,
$$

be an irreducible plane $n$-ic; then the discriminant $\Delta$ of $C_{n}$ is a homogeneous polynomial of degree $3(n-1)^{2}$ in the coefficients. If

$$
a_{\alpha_{1} \beta_{1} \gamma_{1}}^{p_{1}} a_{\alpha_{2} \beta_{2} \gamma_{2}}^{p_{p_{1}}} a_{\alpha_{z} \beta_{3} \gamma_{z}}^{p_{1}} \cdots a_{\alpha_{k} \beta_{k} \gamma_{k}}^{p_{k}},
$$

$p_{1}+p_{2}+p_{8}+\cdots+p_{k}=n$, denotes the general term of $\Delta$, the indices and exponents of the $a$ 's have the property that each term of $\Delta$ has the same weight $n(n-1)^{2}$ with respect to each of the three indices, $\ddagger$ i. e.

$$
\begin{aligned}
\alpha_{1} p_{1}+\alpha_{2} p_{2}+\cdots+\alpha_{k} p_{k} & =\beta_{1} p_{1}+\beta_{2} p_{2}+\cdots+\beta_{k} p_{k} \\
& =\gamma_{1} p_{1}+\gamma_{2} p_{2}+\cdots+\gamma_{k} p_{k}=n(n-1)^{2} .
\end{aligned}
$$

III. THE $n$-IC THROUGH THE PROJECTIONS FROM A GENERIC POINT IN SPACE OF $N=n(n+3) / 2$ POINTS UPON A PLANE

Following a procedure similar to that of the paper mentioned above, let $A_{i}\left(a_{1}^{i} a_{2}^{i} a_{3}^{i} a_{4}^{i}\right), i=1,2, \cdots, N$, be the fixed points and $P\left(x_{1} x_{2} x_{3} x_{4}\right)$ the center of projection, so that the projection of $A_{i}$ upon $x_{4}=0$ is

- Presented to the Society, May 1, 1926; received by the editors in December, 1925.

† These Transactions, vol. 27 (1925), pp. 270-278.

‡ B. Bauer, Vorlesungen uber Algebra, 1910, pp. 88-97. 
$A_{i}^{\prime}\left(a_{k}^{i} x_{4}-a_{4}^{i} x_{k}\right), k=1,2,3$, or using cartesian coördinates for the points in space, $A_{i}^{\prime}\left(x-a_{i}, y-b_{i}, z-c_{i}\right)$. The plane $n$-ic through the points $A_{i}^{\prime}$ is in determinant form

(4)

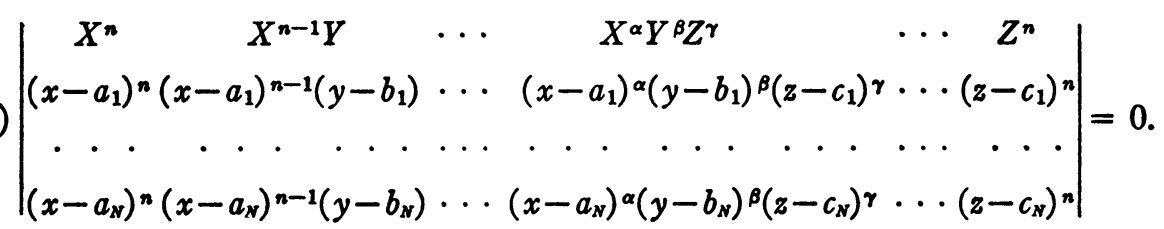

Thus, denoting the reduced expression by

$$
\sum a_{\alpha \beta \gamma} X^{\alpha} Y^{\beta} Z^{\gamma}=0,
$$

and following a procedure similar to that of my previous paper, from (4) it is seen that the coefficient $a_{\alpha \beta \gamma}$ is of degree $\frac{1}{6} n(n+1)(n+2)-\alpha$ in $x$, $\frac{1}{6} n(n+1)(n+2)-\beta$ in $y$, and $\frac{1}{6} n(n+1)(n+2)-\gamma$ in $z$.

\section{Curves with singularities}

We now construct the discriminant of (5) which has the form expressed by (2). According to the result just stated in the foregoing sections, its degree in $x$ will be that of $a_{\alpha_{1} \beta_{1} \gamma_{1}}^{p_{1}} \cdot a_{\alpha_{2} \beta_{2} \gamma_{2}}^{p_{2}} \cdots$ in $x$, or

$$
\sum\left[\frac{1}{6} n(n+1)(n+2)-\alpha_{i}\right] p_{i}=\frac{1}{6} n(n+1)(n+2) 3(n-1)^{2}-n(n-1)^{2} .
$$

As this may be written in the form

$$
\frac{1}{3} 3(n-1)^{2} n \frac{(n+3)}{2}
$$

it follows that the degree in $x$ (and also in $y$ and $z$ ) is equal to one third times the degree of the discriminant of a ternary n-ic, times the order of the n-ic, times the number of points necessary to determine a plane $n$-ic.

The expression $\Delta$ in its reduced form is a polynomial in $x, y, z$ of this same degree, since $(y=0, z=0)$, which is a generic line with respect to $\Delta=0$, cuts the surface $\Delta=0$ in as many points as there are roots in the resulting equation $\Delta(x, 0,0)=0$. As the coefficients of $\Delta$ contain each triple $x-a_{i}, y-b_{i}, z-c_{i}$ homogeneously of degree $n$, the fixed points $A_{i}$ are multiple points of order $3 n(n-1)^{2}$ of $\Delta=0$. Moreover a point on the join of two $A_{i}$ 's is the vertex of $3(n-1)^{2} n$-ic cones with singular generatrices. Hence the

THEOREM. The locus of vertices of $n$-ic cones with nodal generatrices through $\frac{1}{2} n(n+3)$ generic points in space is a surface of order $\frac{1}{2}(n-1)^{2} n^{2}(n+3)$, which has each of these points as a multiple point of order $3 n(n-1)^{2}$, and each join of two of these points as multiple line of order $3(n-1)^{2}$. 


\section{Examples}

In case of five points, $n=2$, the order of $\Delta=0$ is $w=10$. This can easily be verified geometrically. The five points in groups of three can be joined by 10 planes. Each plane with any plane through the join of the two remaining points forms a degenerate cone through the five points. Thus the first of these two planes may be considered as the locus of vertices of $\infty^{1}$ degenerate cones. The same is true for each of the remaining 9 planes. Through the join of two of the five points pass three of these planes, so that this join is a triple line of the surface. Through each of the five points there are six of these planes, so that the point is six-fold for the surface. These numbers are in agreement with the numbers in the general case.

Cubic cones. For $n=9$, we may restate the

THEOREM. The locus of vertices of cubic cones with singular generatrices through 9 points is a surface of order 108, with 36-fold points at these points and with each join of two as a 12-fold line.

A plane $p$ through three of the 9 points cuts the surface in a plane curve of order 108. But each of the three sides of the triangle determined by the three points must be counted 12 times in the intersection, so that the curve of intersection proper is of order $108-48=60$ which has 12 -fold points at each of the $(6 \cdot 5) / 2=15$ points of intersection of the joins of the six points outside of $p$ with $p$. The Weddle surface determined by the six points cuts $p$ in a quartic which passes singly through each of the 15 points. This is a component part of the curve of order 60 because each point of this quartic is the vertex of a quadric cone which together with $p$ forms a degenerate cubic cone. The residual curve of vertices in $p$ of non-degenerate singular cubic cones is therefore a curve of order 56 with 11 -fold points at the 15 points.

In the previous paper the existence of a curve $R_{12}$ is established from whose points the 9 points are projected into 9 base points of a pencil of cubics. There are 12 singular cubics in every pencil. Consequently every point of $R_{12}$ is the vertex of 12 cubic cones with singular generatrices. From this it follows that the surface of order 108 has $R_{12}$ as a 12-fold singular line.

UNIVERSITY OP ILLINOIS, URBANA, ILI. 